\title{
A Comparative Analysis of Digital Filtering for Seismic Signals from Adana, Van, and Bingol Earthquakes in Turkey
}

\author{
Samer Al-Khateeb ${ }^{1, a}$, Engin Mendi ${ }^{1,2, b}$, and Coskun Bayrak ${ }^{1, c}$ \\ ${ }^{1}$ Computer Science Department, University of Arkansas at Little Rock, AR, USA \\ ${ }^{2}$ Computer Engineering Department, KTO Karatay University, Konya, Turkey \\ asxalkhateeb@ualr.edu, bengin.mendi@karatay.edu.tr, ccxbayrak@ualr.edu
}

Keywords: PGA, PGV, PGD, Butterworth, Bessel, Chebyshev (type I), SAC, Acausal filters.

\begin{abstract}
This paper includes a comparative application of the digital filters on data processing for the strong motion data recorded during earthquakes. Three types of digital, acausal filters (designed by Butterworth, Bessel, and Chebyshev type I) with $\mathrm{N}=4$ were used to remove the unwanted noise. The data has been collected from three different stations located in Turkey (Adana, Van, and Bingol) that are working with a digital type of seismograph (Guralp cmg5td and SigSasmach). Those stations are close to the earthquake events with different moment magnitudes $\mathrm{Mw}=4.5,3.8$, and 3.3. The SAC software tool has been used to evaluate digital filter influence on time series (acceleration, velocity, and displacement). This has been done by measuring and comparing some strong motion parameters (peak ground acceleration, peak ground velocity, and peak ground displacement).
\end{abstract}

\section{Introduction}

Natural disasters are important phenomena in human life. Hurricanes, earthquakes, severe weather, floods, and volcanic eruptions, all can cause property damage or loss of life. Therefore, it is important to study these disasters, and know how to deal with them to minimize the damage they can cause to humans. One important phenomenon is an earthquake, which is the focus of this research. Earthquakes are measured by their magnitude and intensity. Both magnitude and intensity are an expression of the amount of energy released when a fault ruptures. Magnitude is a measurement of the energy released by an earthquake. Intensity is the strength of seismic shaking at a given location (how much damage the shaking cause). These magnitudes and intensities can be measured by seismic instruments, like accelerographs, that can record signals caused by the shake of the Earth. There are two types of accelerographs: Analog (most widely used seismograph SMA-1), and Digital accelerographs (most widely used Guralp cmg5td). In order to use these signals, data processing needs to be applied. After that, strong motion parameters (such as PGA, PGV, and PGD) can be evaluated.

This paper focuses on the processing of two major objectives for strong motion data: (1) Instrument correction to the response of the strong motion data and (2) Noise reduction of the recorded strong motion data. The recorded raw data have to be preprocessed by seismologists before any seismological or engineering analysis can be conducted. There are many different organizations around the world that use different procedures in data processing. In the United States, three major agencies (CSMI (California Strong-Motion Instrumentation Program), PEER (Pacific Earthquake Engineering Research Centre) and USGS (US Geological Survey)) process records under different signal processing techniques for example:

- Instrument Correction.

- Baseline Correction.

- High-Frequency Filtering.

- Computation of Response Spectra.

- High-Pass Filtering. 
In order to apply these signal processing techniques there are many software packages that can be used in the digital seismology field. Some of these widely used packages are:

- SeismoSignal.

- PREPROC.

- PITSA.

- BAP.

- MATLAB.

- SAC (Seismic Analysis Code) [1].

Strong motion data can be processed using different causal or acausal filters. The choice of the actual generic filter (Bessel, Butterworth, Chebyshev, Ormsby, or elliptical) is not as important as the choice of the right parameters, and the choice whether this filter is causal or acausal filter. Many recent researches investigated whether causal or acausal filters should be used to process the strong motion data. Causal filters "result in phase shifts in the record" and signal distortions while, acausal filters "do not produce any phase distortion in the signal" (zero phase shift) [2].

\section{Background}

For the focus of this research, data is collected from earthquakes that occurred in three Turkish cities (Adana, Van, and Bingol) shown in Fig. 1. These events where recorded by three stations using digital accelerographs (Guralp cmg5td and SigSasmach) [3]. The data under investigation is shown in Table 1. These data will be filtered using Acausal band pass filters designed by Butterworth, Bessel, and Chebyshev (type I). Since this data is recorded using digital instruments, there is no need to apply instrument corrections [2].

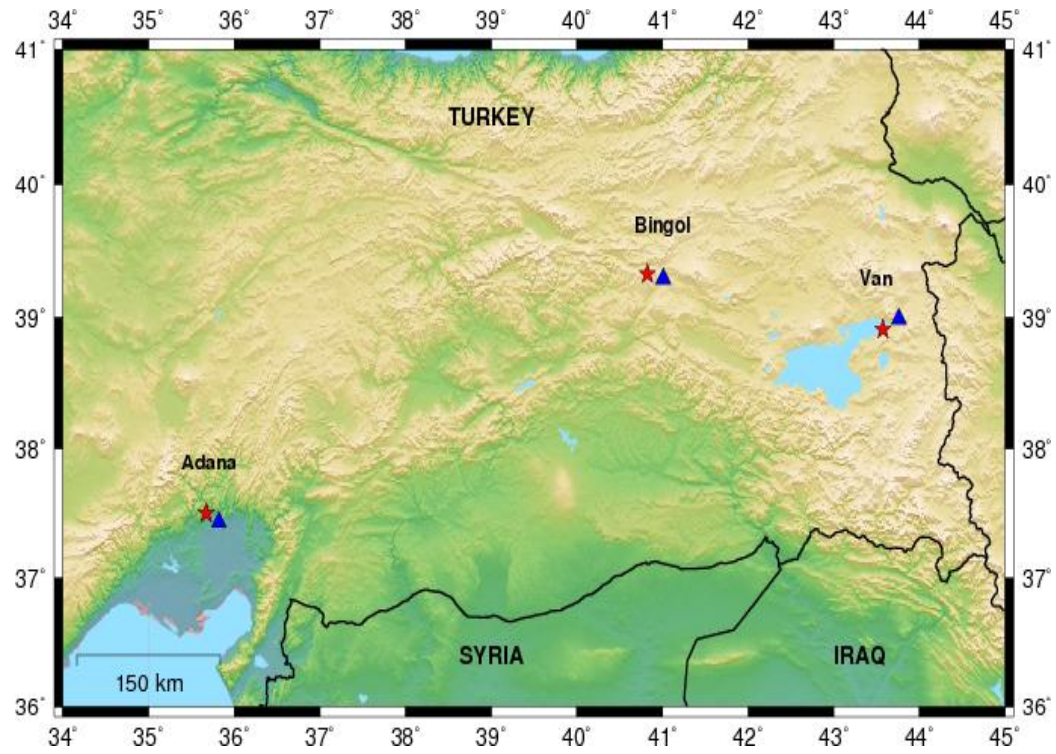

Fig. 1. Location of the stations and events under investigation (red star denoted to an event and blue triangle denoted to a station).

Table 1. Stations and Data under Invistigation

\begin{tabular}{|l|l|l|l|l|l|}
\hline Stations ID & $\begin{array}{l}\text { Station } \\
\text { Coordinates }\end{array}$ & Event (date) & $\begin{array}{l}\text { Epicenter } \\
\text { Coordinates }\end{array}$ & $\begin{array}{l}\text { Magnitude } \\
{[\mathrm{ML}]}\end{array}$ & $\begin{array}{l}\text { Earthquake Depth } \\
{[\mathrm{Km}]}\end{array}$ \\
\hline 6506 & $39.01963 \mathrm{~N}$ & $11 / 21 / 2011$ & $\begin{array}{l}38.68850 \mathrm{~N} \\
43.22300 \mathrm{E}\end{array}$ & 4.5 & 17.88 \\
\hline 1206 & $\begin{array}{l}43.33797 \mathrm{E} \\
41.29345 \mathrm{~N}\end{array}$ & $08 / 20 / 2012$ & $\begin{array}{l}39.32970 \mathrm{~N} \\
40.82330 \mathrm{E}\end{array}$ & 3.8 & 5 \\
\hline 0122 & $\begin{array}{l}37.00883 \mathrm{E} \\
35.43390 \mathrm{~N}\end{array}$ & $09 / 15 / 2012$ & $\begin{array}{l}37.50400 \mathrm{~N} \\
35.67350 \mathrm{E}\end{array}$ & 3.3 & 20.22 \\
\hline
\end{tabular}


In [1], data that was collected by three stations situated in Kusadasi, Izmit and Dinar in Turkey is investigated. These stations were operating with an analogue type of seismograph known as SMA-1. The data of these stations were collected from sources close to the earthquakes (the distance to the epicenter $>20 \mathrm{~km}$ ). These events with various magnitudes $\mathrm{Mw}=7.4,6.4$, and 3.8 have been tested. Evaluation of the type of digital filters influence, on time series (acceleration, velocity, and displacement) for some strong motion parameters (peak ground acceleration, velocity, and displacement) has been conducted.

There is standard and non-standard noise that can come with digitized records from analog instruments. Standard noise can be eliminated or reduced by applying processing procedures. Non-standard noise should be identified and eliminated, to the possible extent, prior to undertaking any processing. One example of the non-standard noise is spurious or 'spikes' (broadband that have energy content at short and long periods). In order to identify this kind of noise, a comparison with the reproduction of the original analog data have to be done. To eliminate this noise, the acceleration ordinates of the spike need to be replaced with the mean of the acceleration for the data points on either side.

Instrument noise is another type of noise that can be found in digitized records. Instrument corrections are not important for digital recordings however, it should be considered for analog data, especially if the engineering application is interested in motion at frequencies greater than $20 \mathrm{~Hz}$. It is advisable that instrument correction should not be considered unless there are compulsory reasons for applying it.

One way to correct signals, which are obtained from both analog and digital accelerographs, is to use baseline correction. Baseline correction can be used to partially eliminate this kind of noise. Accelerographs signals, without baseline correction, can result in unreal velocities and displacements. Baseline amendments are high-pass filters of obscure frequency characteristics. There are two ways to mitigate these problems and to use baseline amendments. One way is a quadratic fit to the velocity, which is a simple form of more complex method that was suggested by Graizer [4]. The second way was suggested by Iwan et al. [5], which is more complicated and needs complex set of baseline shifts. These baseline shifts are chosen in a way that one of them is in between $t 1$ and $t 2$, while the other one is after $t 2$. This amendment is applicable to any data. The benefit of it is that the velocity will fluctuate around zero (actual restriction), however, this amendment seeks selection of $t 1$ and $t 2$, without reasonable reason for selecting these times (for instance, according to the acknowledgement of a certain instrument). Another way to correct signal and reduce noise in accelerographs is to use filters. For acausal filters the number of passes is 2 and for causal filters the number of passes is 1 . Acausal filters are much better than causal filters in processing strong motion data because "they do not produce any phase distortion in the signal". Phase distortion should be avoided because it makes some parts of the output signal out of phase with the rest of the output. The filter order affects the band pass filter curve sharpness. The rolls off/ramp sharpness increase with higher filter order [2].

\section{Methodology}

The overview of the system that has been developed is shown in Fig. 2. It is designed to be a three-phased system: data collection, pre-processing, and data processing.

Data Collection. There were three events that occurred in three Turkish cities (Adana, Van, and Bingol) in 2011 and 2012, and these events where recorded by three stations. Each station recorded three components for the same earthquake. The recorded data sent into an online database, where it can be easily access and download in a text file format as shown in Fig. 2.

Pre-Processing. The text files separated into three components using Microsoft EXCEL. Then these data were converted using MATLAB into an ASCII files to be used by SAC software as in Fig. 2. After the data has been converted into SAC format, all data processing has been performed using SAC software. SAC is an interactive command driven program developed by the University of California. 


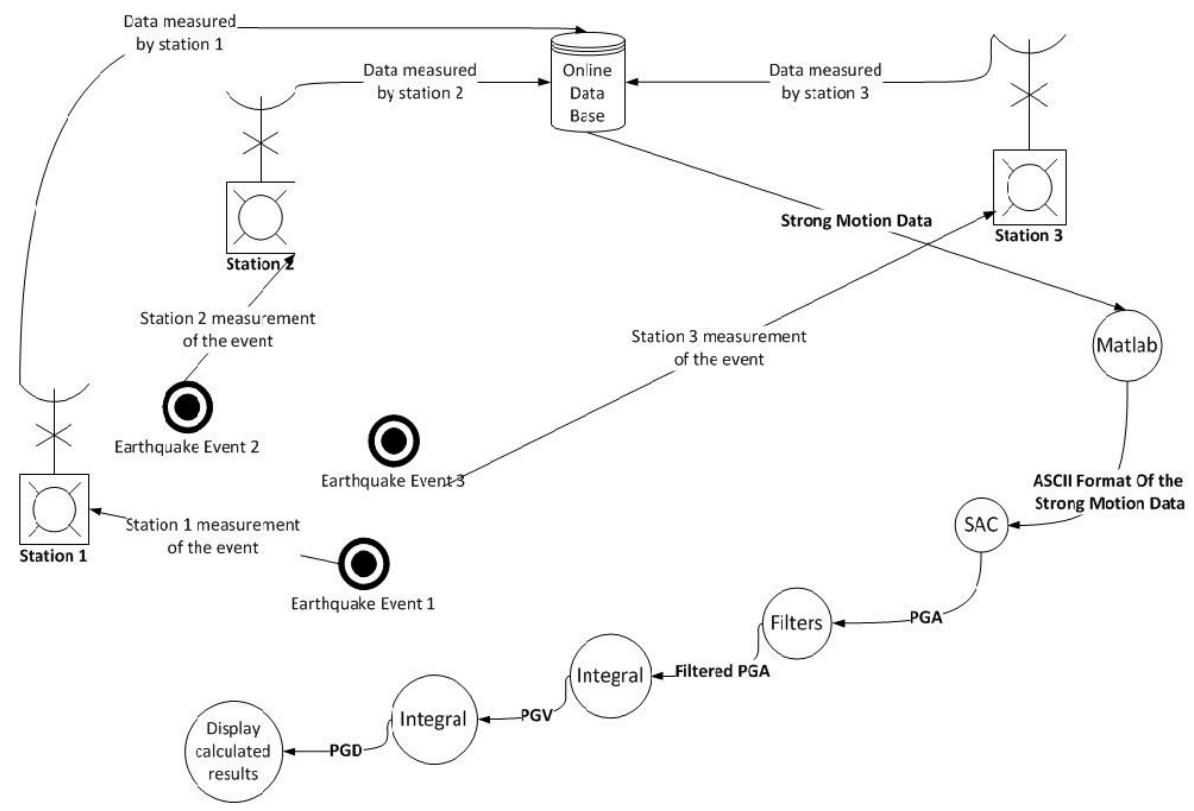

Fig. 2. The overview of the system.

Data Processing. The flow chart for the data processing that has been performed using SAC software is given in Fig. 3. The strong motion data is integrated to get the velocity data, and then the velocity data is integrated to get the displacement data. Then, the acceleration, velocity and displacement has been filtered using Butterworth, Bessel, and Chebyshev type I filters. Finally, the PGA, PGV, and PGD parameters are calculated [2].

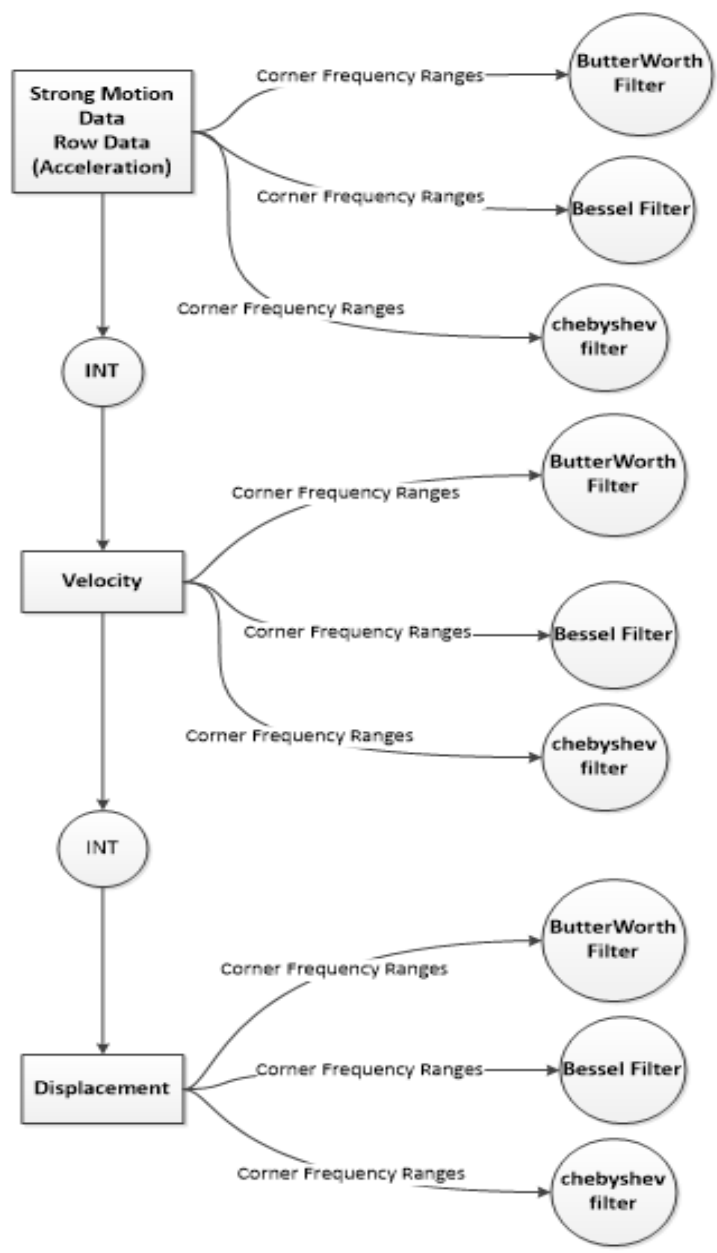

Fig. 3. Data processing. 
Each signal was represented in three components: two horizontal and one vertical component. All the filters and data processing were applied to the vertical component, since this usually has a lower signal to noise ratio than the horizontal components, particularly in the long-period range. Choosing three components from two different stations for the same event and the graphs looks the same; confirm the selection of the appropriate filter parameters. In order to obtain the period of time over which the data can be used, the header file of each data set need to be checked [2].

The results of applying the filters are shown in Table 2, Table 3, and Table 4, respectively.

Table 2. Adana Vertical Component

\begin{tabular}{|c|c|c|c|c|c|c|}
\hline Parameters & Raw & F1 [Hz] & F2 [Hz] & Butterworth & Bessel & Chebyshev \\
\hline PGA & 6.369 & 10.5 & 14.5 & 3.60214 & 3.53963 & 1.15404 \\
\hline PGV & 0.101 & 10.5 & 14.5 & 0.0427994 & 0.0397716 & 0.0138857 \\
\hline PGD & 0.035 & 10.5 & 14.5 & $5.06232 \mathrm{E}-4$ & $4.7648 \mathrm{E}-4$ & $1.7062 \mathrm{E}-4$ \\
\hline
\end{tabular}

Table 3. Van Vertical Component

\begin{tabular}{|c|c|c|c|c|c|c|}
\hline Parameters & Raw & F1 [Hz & F2 [Hz] & Butterworth & Bessel & Chebyshev \\
\hline PGA & 3.801 & 1.5 & 5.5 & 2.5294 & 2.298 & 0.786451 \\
\hline PGV & 0.127317 & 1.5 & 5.5 & 0.105874 & 0.0941 & 0.0322474 \\
\hline PGD & 0.36456 & 1.5 & 5.5 & $6.5723 \mathrm{E}-3$ & $6.0144 \mathrm{E}-3$ & $1.83592 \mathrm{E}-3$ \\
\hline
\end{tabular}

Table 4. Bingol Vertical Component

\begin{tabular}{|c|c|c|c|c|c|c|}
\hline Parameters & Raw & F1 [Hz] & F2 [Hz] & Butterworth & Bessel & Chebyshev \\
\hline PGA & 9.8011 & 7.98 & 11.98 & 5.3265 & 5.03006 & 1.82949 \\
\hline PGV & 0.16052 & 7.98 & 11.98 & $8.449 \mathrm{E}-2$ & $7.866 \mathrm{E}-2$ & $2.89168 \mathrm{E}-2$ \\
\hline PGD & 0.04533 & 7.98 & 11.98 & $1.2903 \mathrm{E}-3$ & $1.2349 \mathrm{E}-3$ & $4.61822 \mathrm{E}-4$ \\
\hline
\end{tabular}

\section{Analysis of Results}

The appropriate velocity and displacement data were acquired using SAC software by taking single and double time integration for the acceleration time histories, respectively. For each event, the time for the maximum peak value to happen in the acceleration record is calculated. Then, the frequency is computed by taking the reverse of that time. Finally, the corner frequency of the filters is calculated by taking the frequency required by the maximum peak value $\pm 2 \mathrm{~Hz}$. Eventually the band pass filter with order $N=4$ is applied, because it is the best choice recommended to apply in [1]. With the corner frequencies, the best waveforms that are very close to the uncorrected one is obtained as shown in Fig. 4, Fig. 5 and Fig. 6, respectively.

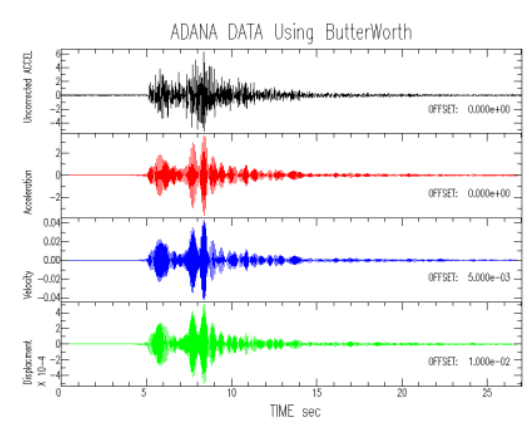

(a)

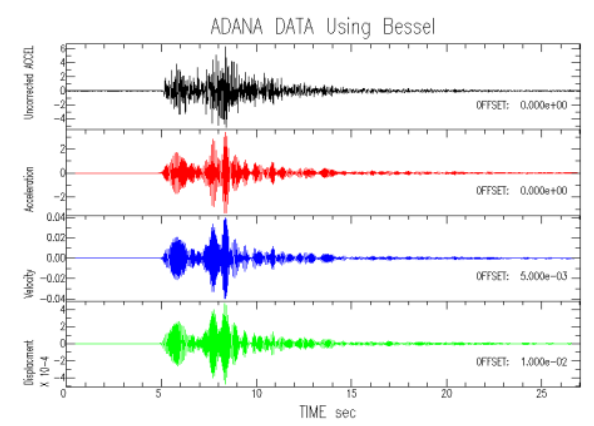

(b)

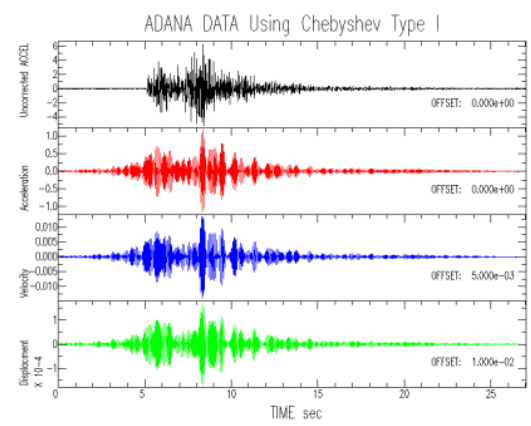

(c)

Fig. 4. Uncorrected ADANA Acceleration, corrected Acceleration, Velocity, and Displacement (UD Component) using band pass filter: Order $=4, \mathrm{f} 1=10.5 \mathrm{~Hz}, \mathrm{~F} 2=14.5 \mathrm{~Hz}$. 


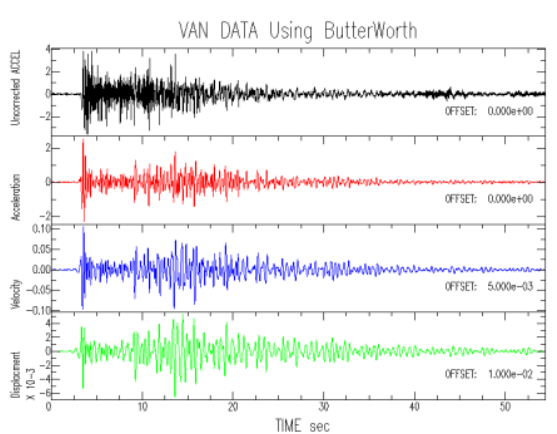

(a)

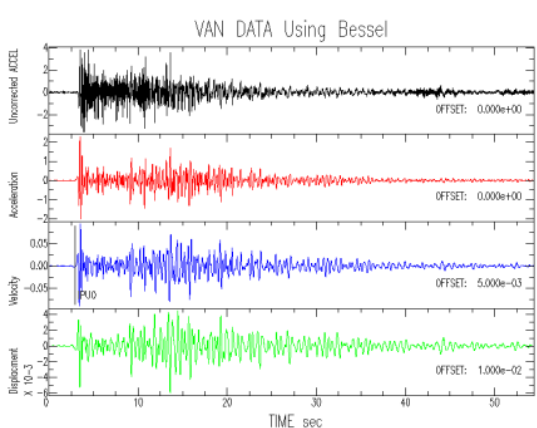

(b)

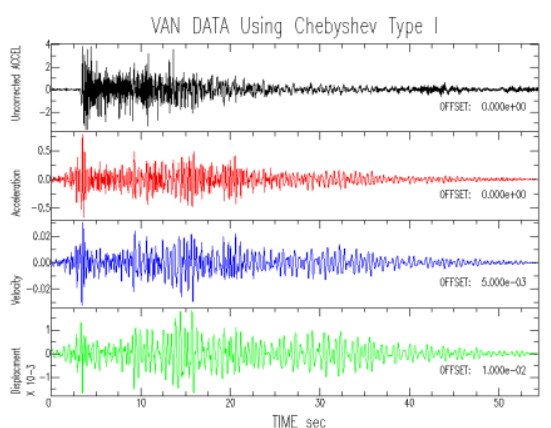

(c)

Fig. 5. Uncorrected VAN Acceleration, corrected Acceleration, Velocity, and Displacement (UD Component) using band pass filter: Order $=4, \mathrm{f} 1=1.5 \mathrm{~Hz}, \mathrm{~F} 2=5.5 \mathrm{~Hz}$.

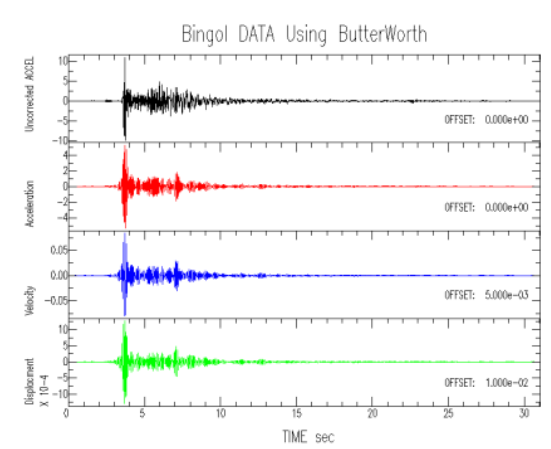

(a)

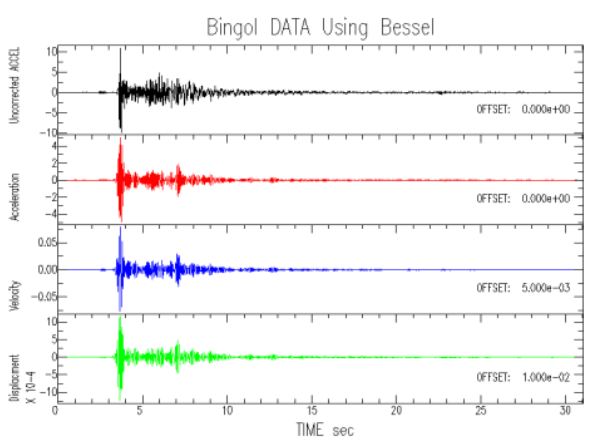

(b)

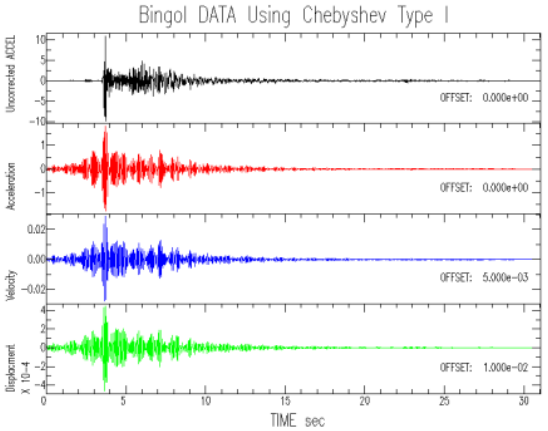

(c)

Fig. 6. Uncorrected BINGOL Acceleration, corrected Acceleration, Velocity, and Displacement (UD Component) using band pass filter: Order $=4$, f1=7.98Hz, F2=11.98 Hz.

As shown in Table 2, Table 3, and Table 4, Butterworth filter absorb the least amount of energy comparing to the other two types of filters (Bessel and Chebyshev Type 1). Using Chebyshev filter, the waveform start to look different than the wave form using the other two filters (Butterworth and Bessel) as can be clearly seen in Fig. 6 (b) and Fig. 6 (c). The mean and trend have been removed from the graphs before further processing of the waveforms.

\section{Conclusion}

Strong motion data always have noise to some degree and to process these data the noise need to be reduced or removed without changing the shape of the waveform abnormally. Also the best filter is the one which absorbs the least amount of energy from the signal. Based on this research two important conclusions can be drawn. First, Butterworth filters absorb the least amount of energy and Chebyshev filters absorb the most, while Bessel filters lay in between. Second, Butterworth and Bessel filters do not change the shape of the waveform so much from the uncorrected one while Chebyshev does change the waveform under the same corner frequency applied.

\section{Acknowledgement}

We would like to thank Dr. Hanan Mahdi and the earthquake center at UALR for providing the equipment and the software that has been used in this research.

\section{References}

[1] G. Mollova, "Effects of digital filtering in Data Processing of Seismic Acceleration Records", EURASIP Journal on Advances in Signal Processing, vol. 2007, Article ID 29502, 2007. 
[2] Boore, D., and J. Bommer. "Processing of Strong-motion Accelerograms: Needs, Options and Consequences." Soil Dynamics and Earthquake Engineering 25.2 (2005): 93-115. 25 Oct. 2004. Web. 10 Feb. 2013.

[3] KyhData V4.0, Strong Ground Motion Database of Turkey. KyhData V4.0, n.d. Web. 08 Mar. 2013. <http://kyhdata.deprem.gov.tr/2K/kyhdata_v4.php?dst=TU9EVUxFX05BTUU9ZXZ0RmlsZS ZNTORVTEVfVEFTSz1zZWFyY2g=>.

[4] Graizer VM. Determination of the true ground displacement by using strong motion records. Izvestiya, Phys Solid Earth 1979; 15: 875-85.

[5] Iwan WD, Moser MA, Peng C-Y. Some observations on strongmotion earthquake measurement using a digital accelerograph. Bull Seismol Soc Am 1985; 75:1225-46.

[6] B. Darragh, W. Silva, and N. Gregor, "Strong motion record processing for the PEER centre", Proceedings of COSMOS Invited Workshop on Strong-Motion Record Processing, Richmond, CA, USA, May 2004.

[7] SAC Tutorial Guide for New Users, SAC Tutorial Guide for New Users. The University of $\begin{array}{lllllll}\text { California, } & 15 & \text { Aug. } & 2006 . & \text { Web. } & 24 & \text { Feb. }\end{array}$ <http://www.iris.edu/software/sac/manual/tutorial.html>. 